\title{
Service and multimedia data transmission in loT networks using hybrid communication devices
}

\author{
Anton Saveliev ${ }^{1, *}$, Dmitry Malov ${ }^{2}$, Michael Tamashakin ${ }^{1}$ and Victor Budkov ${ }^{1}$ \\ ${ }^{1}$ SPIIRAS, Laboratory of Speech and Multimodal Interfaces, 199178 Saint-Petersburg, 14-th line of V.I., 39, Russia \\ ${ }^{2}$ SUAI, Department of Electromechanics and Robotics, 190000 Saint-Petersburg, Bolshaya Morskaya str., 67, Russia
}

\begin{abstract}
Employment of various protocols and technologies in IoT networks leads to the lack of module unification and increase in incompatible technical solutions. Modern IoT networks are not designed for streaming audio/video data, so their application field is limited. Also, modern IoT networks should have connection areas for devices transferring data to the Internet, and consider hardware and software specific characteristics of these devices. We offer one-size-fits-all solution for organization of IoT network, using hybrid modules. These devices provide flexibility, scalability, energy efficiency and multi-use of network for the transfer of various types of data. This approach takes into account software and hardware features of the devices used for data transmission in IoT networks, which helps to automate connecting the modules chosen by user.
\end{abstract}

\section{Introduction}

IoT networks are one of the most perspective solutions for increasing the level of automation in different areas of human activity. Currently, IoT technologies are being successfully implemented not only in service business, but also in real sector of economy, e.g. mining and oil \& gas industry. One of the most popular application of IoT technologies is "Smart Home" systems, which allow simplifying human interaction with systems and devices of their house $[1,2]$. Also, such technologies are widely used in medicine [3,4] for extracting, storing and transmitting information about indicators of human vital functions.

Analysis of big data accumulated by the IoT devices allows formulating not only analytical reports, but also recommendations for decision makers [5,6]. Moreover, analytical data collected by the IoT systems can be useful during operation and testing of various devices. For example, in [7], a method for monitoring structural state of air and space craft using wireless sensor networks is presented. The paper discusses algorithm of data transmission using wireless IoT networks organization technology ZigBee. Also, the model of wireless sensor network consisting of gateway unit, routing device and motes (autonomous computational devices) with sensors is described.

Apart from information transmission, IoT networks are able, in particular, to simplify problem solution for indoor localization and navigation of autonomous mobile objects (users, robotics platforms, etc.). For example, paper [8] discusses the combined method for mobile object localization and improving its indoor positioning using wireless technologies. The current location of a person is determined using embedded sensors of his ther smartphone. The authors consider technologies sustained by the majority of modern mobile devices: accelerometer, gyroscope, magnetometer, proximity sensor, luminance sensor, as well as Wi-Fi, Bluetooth, GSM, NFC. The best solution, in authors' opinion, is using the Bluetooth beacon that operates according to the iBeacon technology. Besides, the conducted research shows the long-term benefits of the combined method.

One of the specific characters of wireless IoT networks is common wireless environment, based on single technology of data transmission, which makes multiple access of "smart devices" to the environment more difficult. In [9], the procedure of access regulation for "smart devices" was presented, which allows taking into account the specific characteristics of heterogeneous traffic service. The presented computation example proves the implementation efficiency of access regulation for decreasing delays in data frame service of highest priority subnets.

\section{Review of popular audio and video codecs}

Devices of IoT system should provide necessary communication channel capacity for transfer of diverse types of data. Codecs are used for more efficient and fast audio/video data transfer; it's possible to reduce amount of transmitted data, using codecs. Let us consider the most popular and widely used codecs. We can determine the minimum parameters of bitrate for IoT system development (Table 1,2). Table 1 shows that the most demanding of network resources codec is MJPEG, so we

\footnotetext{
* Corresponding author: saveliev@iias.spb.su
} 
set its bitrate as a minimum required network bandwidth for video data transfer.

Table 1. Bitrate of popular video codecs

\begin{tabular}{|c|c|c|c|}
\hline Codec & H.264 & MJPEG & VP8 \\
\hline $\begin{array}{c}\text { Minimum } \\
\text { bitrate, Kbps }\end{array}$ & 736 & 17800 & 11064 \\
\hline
\end{tabular}

Table 2. Bitrate of popular audio codecs

\begin{tabular}{|l|l|l|l|l|l|l|}
\hline Codec & MP3 & FLAC & ALAC & Opus & iSAC & iLBC \\
\hline $\begin{array}{l}\text { Minimum } \\
\text { bitrate, } \\
\text { Kbps }\end{array}$ & 320 & 1200 & 256 & 510 & 56 & 16 \\
\hline
\end{tabular}

The bitrates of the most popular audio codecs are presented in Table 2. The most resource-consuming codec is FLAC, so we set its bitrate as a minimum required network bandwidth for audio data transmission.

\section{Key features of modules for data transfer in loT networks}

To select the appropriate communication devices, consider the key features of popular wireless technologies for IoT system implementation (Table 3).

Table 3. Main technologies for IoT-networks creation

\begin{tabular}{|c|c|c|c|c|c|}
\hline Features & $\begin{array}{c}\text { ZigBe } \\
\mathrm{e}\end{array}$ & $\begin{array}{c}\text { Z- } \\
\text { Wave }\end{array}$ & Insteon & $\begin{array}{c}\text { Bluetooth } \\
4.0 \\
\end{array}$ & Wi-Fi \\
\hline $\begin{array}{l}\text { Frequency, } \\
\quad \mathrm{GHz}\end{array}$ & 2,4 & $\begin{array}{c}0,868- \\
0,908\end{array}$ & $\sim 0,915$ & 2,4 & 2,4 \\
\hline $\begin{array}{c}\text { Bandwidth, } \\
\text { Kbps }\end{array}$ & $\begin{array}{l}\text { Up to } \\
250\end{array}$ & $\begin{array}{c}\text { Up to } \\
100\end{array}$ & $1,2-180$ & $\begin{array}{l}\text { Up to } \\
1000\end{array}$ & $\begin{array}{c}\text { Up to } \\
300000 \\
\end{array}$ \\
\hline $\begin{array}{c}\text { Signal } \\
\text { distance } \\
\text { indoor, } \mathrm{m}\end{array}$ & $10-20$ & $10-30$ & Up to 25 & Up to 60 & $\begin{array}{l}\text { Up to } \\
200\end{array}$ \\
\hline $\begin{array}{c}\text { Max } \\
\text { number of } \\
\text { devices }\end{array}$ & 65536 & 232 & $\begin{array}{c}\text { Not } \\
\text { limited }\end{array}$ & $\begin{array}{c}8 \text { devices } \\
\text { for } 1 \\
\text { piconet, } \\
\text { max } 10 \\
\text { piconets }\end{array}$ & 30 \\
\hline Modes & $\begin{array}{c}\text { Client, } \\
\text { access } \\
\text { point } \\
(\mathrm{CC} 25 \\
30)\end{array}$ & $\begin{array}{c}\text { Client, } \\
\text { access } \\
\text { point }\end{array}$ & $\begin{array}{l}\text { Network } \\
\text { made up } \\
\text { of } \\
\text { independ } \\
\text { ent } \\
\text { devices }\end{array}$ & $\begin{array}{c}\text { Client, } \\
\text { access } \\
\text { point }\end{array}$ & $\begin{array}{c}\text { Client, } \\
\text { access } \\
\text { point, } \\
\text { client } \\
\text { and } \\
\text { access } \\
\text { point }\end{array}$ \\
\hline $\begin{array}{c}\text { Number of } \\
\text { connected } \\
\text { devices }\end{array}$ & 8 & 232 & $\begin{array}{c}\text { Not } \\
\text { limited }\end{array}$ & 7 & 30 \\
\hline
\end{tabular}

Modern IoT systems can be implemented in many ways, but it is necessary to determine the set of data transmission technologies for unification of the development process. According to the characteristics considered above and the analysis of publications, the most perspective technologies for the IoT systems implementation are Wi-Fi and Bluetooth. First, consider their most popular and affordable embedded hardware solutions. A set of Bluetooth modules is presented in Figure 1: a, b, c. These modules can interact with a sensor or a microcontroller by means of the UARTinterface technology.

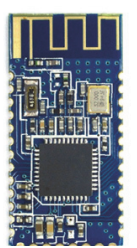

a) HM-10

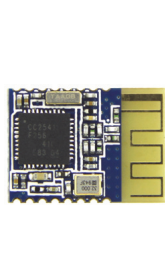

b) HM-11

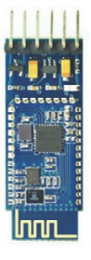

c) $\mathrm{HM}-12$

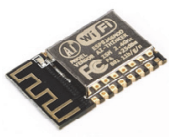

d) ESP8266

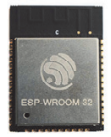

e) ESP32
Fig. 1. Bluetooth and Wi-Fi modules for IoT systems.

The HM-10 and HM-11 modules are low-powered, which makes it possible to use them in systems with low energy consumption. The effective radius of action of these modules is $60 \mathrm{~m}$ outdoors. The modules are small in size and weight and have preinstalled microcontroller software. The HM-12 module has the effective radius of action of up to $100 \mathrm{~m}$ outdoors. Moreover, it has lower energy consumption, compared with other models. All modules discussed above are compatible with Bluetooth 3.0 and 2.1 technologies, so it is possible to use them with the modules of previous versions: HC-05, HC-06, and HC-07. The main characteristics of the HM-10, HM11 and HM-12 modules are presented in Table 4.

Table 4. Main characteristics of HM-10, HM-11, HM-12 modules

\begin{tabular}{|c|c|c|c|}
\hline Name & HM-10 & HM-11 & HM-12 \\
\hline $\begin{array}{c}\text { Bluetooth } \\
\text { version }\end{array}$ & $4.0 \mathrm{BLE}$ & $4.0 \mathrm{BLE}$ & $\begin{array}{c}4.0 \mathrm{EDR} \text { and } \\
\text { BLE }\end{array}$ \\
\hline $\begin{array}{c}\text { Operating } \\
\text { voltage }\end{array}$ & $3.3 \mathrm{~V}$ & $3.3 \mathrm{~V}$ & $3.3 \mathrm{~V}$ \\
\hline $\begin{array}{c}\text { Bitrate. } \\
\text { bps }\end{array}$ & 9600 & 115200 & 115200 \\
\hline Sensibility & $\begin{array}{c}\leq-84 \mathrm{dBm} \\
\text { on } 0.1 \%\end{array}$ & $\begin{array}{c}\leq-84 \mathrm{dBm} \\
\text { on } 0.1 \%\end{array}$ & $\begin{array}{c}\leq-84 \mathrm{dBm} \\
\text { on } 0.1 \%\end{array}$ \\
\hline
\end{tabular}

The Table 4 shows that the Bluetooth 4.0 technology is able to provide transmission of text data packages with low energy consumption, but its bitrate and bandwidth are not enough for audio/video data transfer. Next, consider the Wi-Fi technology that is able to provide enough bandwidth for multimedia streaming.

Apart from the Bluetooth modules, a range of widely used ESP modules can be implemented in IoT systems for data transmission. The ESP8266 module (Figure 1, d) operates with the UART-interface that simplifies the process of connection to a microcontroller or other periphery devices.

The module supports two operational modes: client and access point. Its maximum radius of connection is $400 \mathrm{~m}$. The module energy consumption is $215 \mathrm{~mA}$ in transmission mode and up to $62 \mathrm{~mA}$ in receiving mode. The operational standard of the module is $802.11 \mathrm{~b} / \mathrm{g} / \mathrm{n}$. Its bitrate is up to $3 \mathrm{Mbps}$ that is enough for sound and image transmission. However, it's impossible to organize full-featured streaming video data transmission due to the limited bitrate.

It is reasonable to solve this problem by using the ESP32 module (Figure 1, e). The ESP32 is a hybrid (WiFi and Bluetooth) chip, developed for using in systems, where the values of energy consumption are needed to be minimal (wearable electronics, «internet of things» etc.). The device has $6 \times 6 \mathrm{~mm}$ size. The 
advantages of this module are: combining Wi-Fi and Bluetooth technologies in one chip, two processor cores and a wide range of peripheries. The ESP32 supports protocol stack of Wi-Fi standards 802.11n and BT4.2, providing the functionality by SPI/SDIO and $\mathrm{I}^{2} \mathrm{C} / \mathrm{UART}$ interfaces. The Espressif ESP 32 chip can operate as a CPU (support for Open CPU) or as a "slave device", controlled by a microcontroller. The maximum power needed for data transmission over Wi-Fi is $19.5 \mathrm{dBm}$ on the bitrate up to $150 \mathrm{Mbps}$. The module supports 3 operational modes: "client", "access point" and "client and access point simultaneously". It is possible to provide multimedia data transmission over Wi-Fi using an ESP32 module.

The Bluetooth technology allows organizing piconets in low energy consumption mode and to transfer text data. Thus, it is possible to distribute the load of various types of data transmission between few network standards and to provide the parallel information transmission. Moreover, the Bluetooth technology allows creating systems for space localization of various objects. One may use the Bluetooth modules from the $\mathrm{HC}$ range for nodes that are not supposed for multimedia streaming.

\section{Mathematical models and algorithms of IoT network functioning}

Consider bitrate-distance dependency and determine maximum distances between the modules for stable data transmission. To determine the bitrate-distance dependency for a Wi-Fi network we use data presented in the documentation of standards IEEE 802.11a and 802.11g.

On the basis of primary analysis of the documentation, we can conclude that a linear regression is adaptable for the experimental data approximation. Let us establish the equation of the linear regression of the form $y=a x+b$, where $b$ is an intercept term, and $a$ is a slope coefficient.

The given data are an array of distance values distance $_{a, g}$ and a bitrate bitrate a $_{a, g}$. The given data for IEEE 802.11a standard are:

distance $_{\mathrm{a}}=[24,45,60,69,76,84,91,100], \mathrm{m}$;

bitrate $_{\mathrm{a}}=[54,48,36,24,18,12,9,6]$, Mbps.

The given data for IEEE 802.11g standard are:

distance $_{\mathrm{a}}=$

[30,53,76,84,100,107,110,114,122,128,134,137], m;

bitrate $_{\mathrm{a}}=[54,48,36,24,18,12,11,9,6,5.5,2,1]$, Mbps.

To determine the coefficients $a$ and $b$ the method of least squares is used. The found linear regression equations for IEEE 802.11a standard data (1) and IEEE $802.11 \mathrm{~g}$ standard data (2) are:

$$
\begin{gathered}
\text { bitrate }_{a}=-0.71 \text { distance }_{a}+74.82, \\
\text { bitrate }_{g}=-0.54 \text { distance }_{g}+72.3 .
\end{gathered}
$$

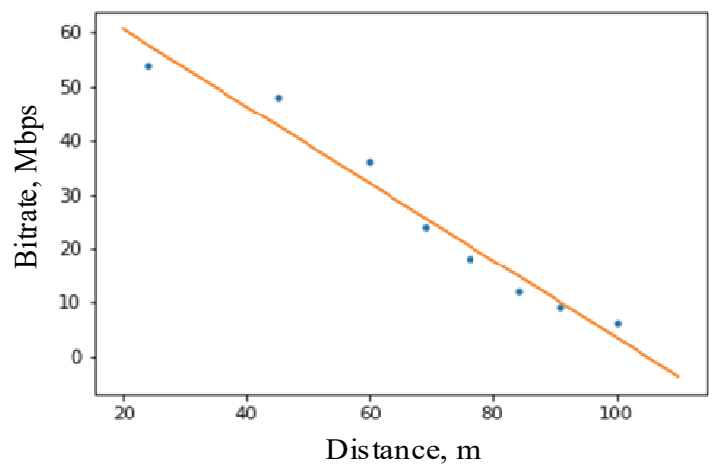

a)

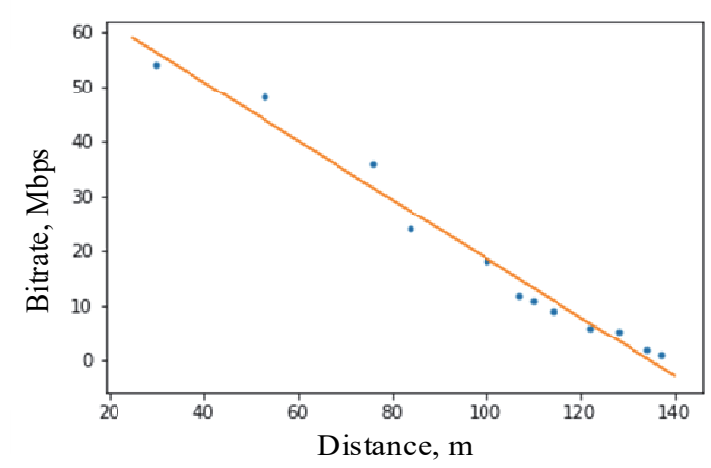

b)

Fig. 2. Bitrate-distance dependency for standards: a) IEEE 802.11a, b) IEEE 802.11b.

As seen from Fig. 2, the considered standards are able to support minimum bitrate $10 \mathrm{Mbps}$ at a distance of up to $80-90 \mathrm{~m}$ indoors without considering any obstacles.

To determine the bitrate-distance dependency for a Bluetooth-network we use formula (3) [17], based on the Friis transmission equation, that gives the power received by one antenna under idealized conditions given that another antenna transmits a known amount of power at some distance [18]:

$$
R S S I=P_{o}-10 n \lg \left(\frac{d}{d_{o}}\right),
$$

where $d$ is the distance between device and transmitter, $\mathrm{m} ; d_{0}$ is the distance between device and point, where signal power $P_{0}$ was measured $(1 \mathrm{~m}) ; n$ is the coefficient of signal energy losses (for air $n=2$ ); RSSI is the received radio signal power level, $\mathrm{dBm}$. To determine the regression equation, we use data from the documentation of the Bluetooth IEEE 802.15.1 standard:

distance $=[0,10,20,40,60,80,100], \mathrm{m}$;

RSSI $=[-35,-38,-36,-37,-45,-48,-43], \mathrm{dBm}$.

Let us transform the RSSI values from $\mathrm{dBm}$ to $\mathrm{W}$ using equation (4):

$$
P_{W}=10^{\left(\left(P_{d B m}-30\right) / 10\right)} .
$$

The obtained dependencies are presented in Figure 2. 
Next, compute the channel capacity using ShannonHartley theorem (5):

$$
C=B \log \left(1+\frac{R S S I}{N}\right)
$$

where $C$ is the channel capacity, $B$ is the channel bandwidth, $N$ is the average power of the noise and interference over the bandwidth. The bitrate-distance dependency for Bluetooth modules is presented in Figure 3.

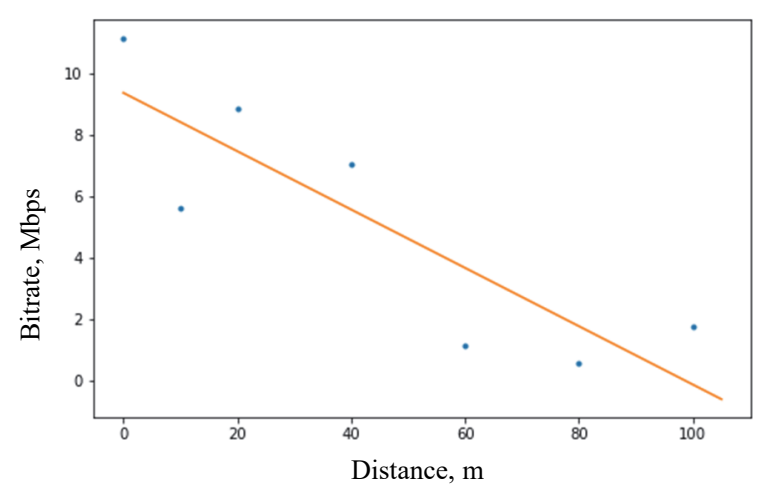

Fig. 3. Bitrate-distance dependency for standard IEEE 802.15.1.

Bluetooth-modules are able to maintain the 3,5 Mbps bitrate at a distance of $65-70 \mathrm{~m}$ (indoors without considering any obstacles). This value is enough for text data transmission. Thus, it is necessary to create an automatic system, which is able to take into account the difference between bitrates considered above. Also, the system should provide the end user with tools for simplified configuration of such hybrid network and selecting a gateway unit and devices. The algorithms developed to achieve this goal are presented in Figures 4 and 5 .

An array of unprocessed devices is fed to the input of the algorithm presented on Figure 4. Next steps are consistently realized for each device in the set. The type of device is extracting. Based on this information, the system determines which devices can be connected with this module. Then, the system is looking for the closest device for the further connection establishment. If effective radiuses of the devices are disjoint, the necessary number of repeaters will be added into the network to provide connection between the devices. For appropriate algorithm operation, it is necessary to check whether any possible connections of the device are left. If there are no available connections left, the device is put in array of inaccessible devices and will be no longer used for searching for new connections. The last step of the algorithm is checking whether there are any unprocessed devices in the system. After the algorithm has done, the IoT system has fully connected devices.

To set a connection to the Internet, the end device data is fed to the input of the algorithm presented in Figure 5. These data are used to create the optimal areas of gateway unit installation or selecting the closest device with necessary type of connection: the user should point a place in the room or a device of the IoT network. Then, the system checks user's input data. If a device of the IoT network is chosen, the algorithm proceeds to the last step. In case of choosing a gateway unit, the system checks whether all the devices are within the gateway's effective radius. If it's not, some repeaters will be added in the system. The connections of these devices are removed and these devices get status "unprocessed". Then, the data about these devices are passed to the input of the algorithm for setting connections between the IoT devices (Fig. 4). The last step is the connection between the end device and the Internet.

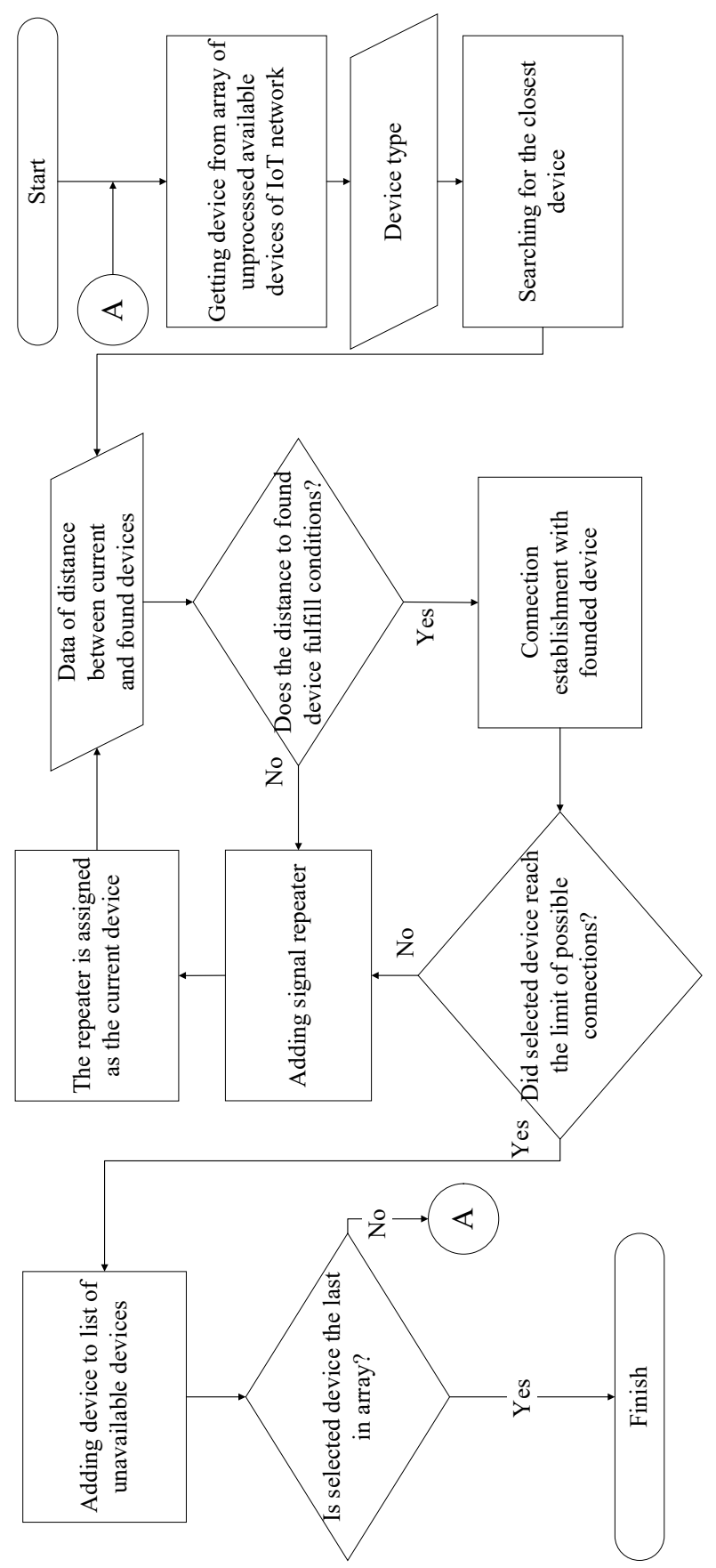

Fig. 4. Algorithm of IoT devices connection.

On the basis of the algorithms discussed above, consider a possible interface of the user-system interaction (Fig. 6). 
At the first step (a), all devices are available to the user (except for gateway unit and repeater) for installation in the room. After the user has located all devices, the system shows devices' effective radiuses (b): the Wi-Fi module has green signal coverage, the Bluetooth module has blue signal coverage.

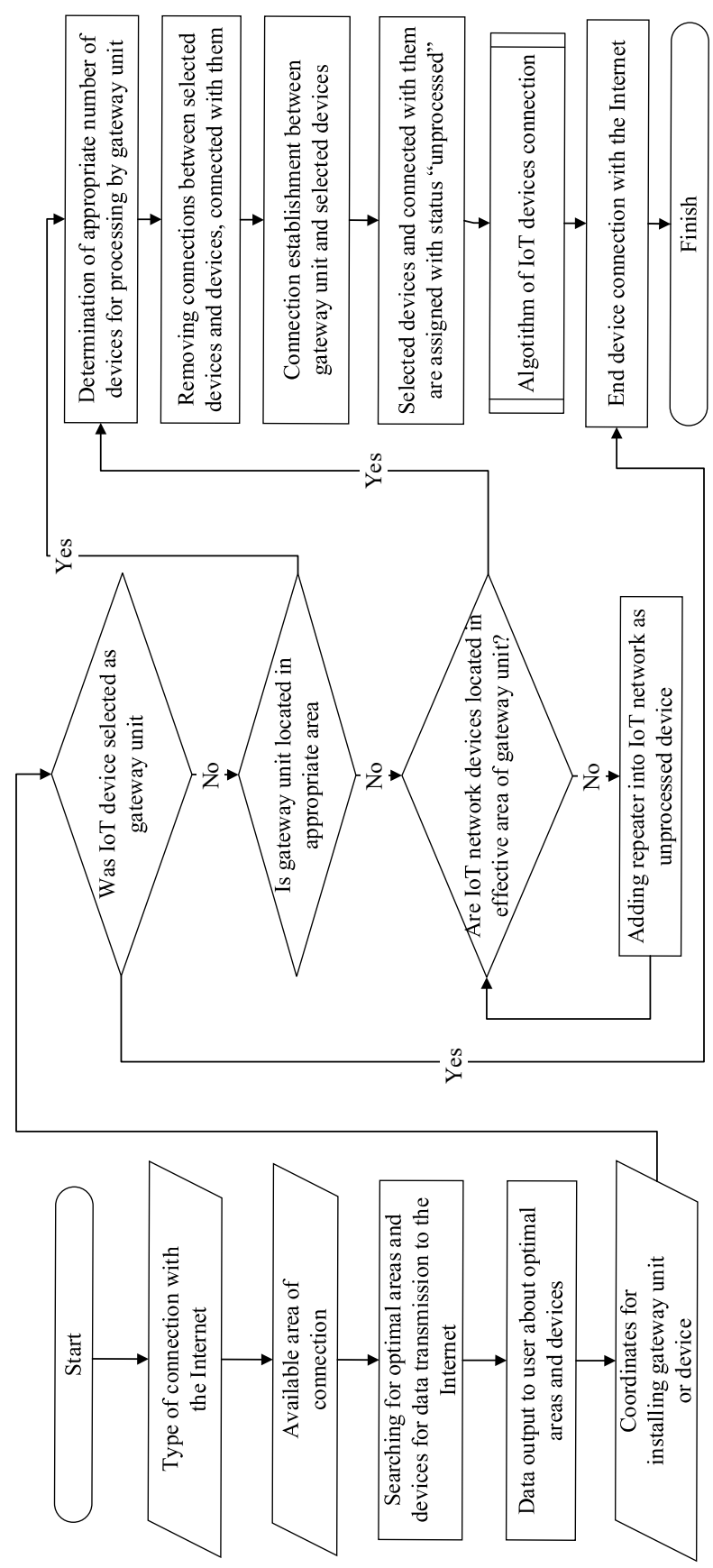

Fig. 5. Algorithm of connection of local IoT network with the Internet.

If user chooses a stand-alone device as a gateway unit, the system offers to install it in the appropriate area ((c): the gateway unit is marked as «GU»). Then the system checks possibility of network organization based on gateway's effective radius. In case it is not enough to cover all devices (d), the system offers to install repeaters where it is necessary ((e): the repeater is marked as $\langle\mathrm{R} »)$ and waits for user confirmation of repeaters installation or changing gateway's location. If user decides to use an already processed device as a gateway unit, the system shows an updated effective radius of the gateway device ((f): the gateway device is marked as «GU»). After all the computations that are needed for organizing the IoT network are done, the system is waiting for user confirmation.

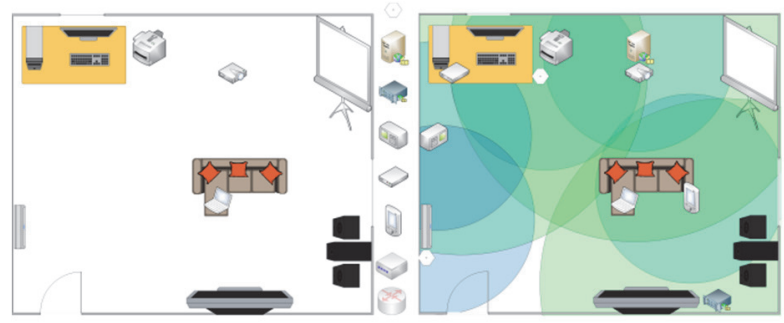

a)

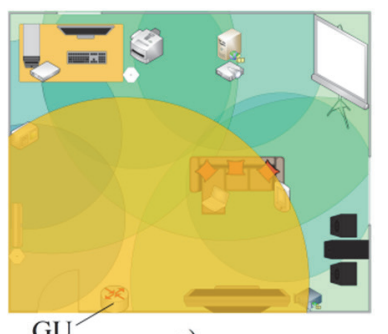

c)

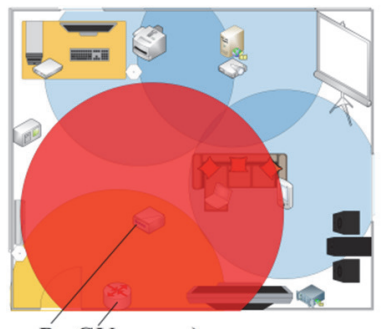

R GU e) b)
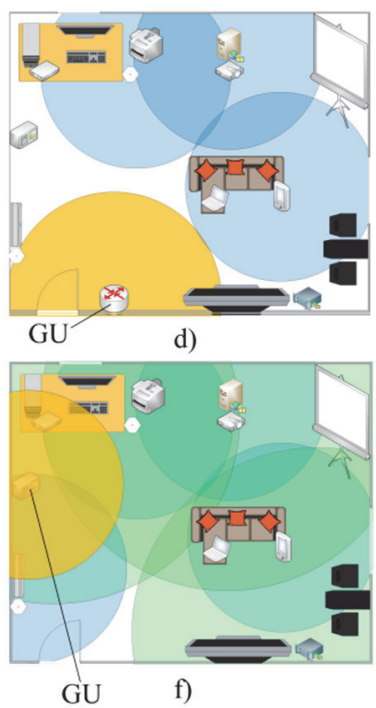

Fig. 6. System interface of hybrid system configuration.

\section{Conclusion}

In the paper the essential components for IoT network's organization were analysed. The parameters of network devices, necessary to provide multimedia data transmission, are found. Based on these parameters, the existing modules able to provide the necessary bitrate of audio/video streaming data transmission were selected. Moreover, the bitrate-distance dependency was analysed for modules working on $\mathrm{Wi}-\mathrm{Fi}$ and Bluetooth technologies. These results were taken as a basis for the algorithms being developed for automatic connection of IoT network's modules. These algorithms allow estimating the distance between devices in the IoT system for providing necessary bitrate of data transmission; adding signal repeaters for the outermost devices' connectivity and organizing connections, which provide data transmission from the IoT network nodes to the Internet. The combination of data transmission technologies provides energy efficiency and cost reduction due to low-load nodes with low bitrate. Also, it supports a high bitrate in the areas where multimedia data transmission is needed. Thus, the hybrid communication modules essentially widen the 
application field of IoT networks and can be integrated in robotics systems [19, 20] for cyber-physical space interaction [21-23] and interaction with users by multimedia information distribution [24, 25].

This work is partially supported by the Russian Foundation for Basic Research (grant No. 15-07-06774-a) and the Council for Grants of the President of Russia (Projects No. MK7925.2016.9).

\section{References}

1. H. Ghayvat, S. Mukhopadhyay, X. Gui, N. Suryadevara, Sensors, 15.5, 10350-10379 (2015)

2. P. Thinagaran, S. K. Datta, C. Bonnet, IEEE 4th Global Conference on Consumer Electronics (GCCE) (2015)

3. Y. Geng, et al., IEEE transactions on industrial informatics, 10.4, 2180-2191 (2014)

4. S.V. Zanjal, G. R. Talmale, Procedia Computer Science, 78, 471-476 (2016)

5. F. J. Riggins, S. F. Wamba, 48th Hawaii International Conference on System Sciences (HICSS), 1531-1540 (2015)

6. B. Payam, A. Sheth, C. Henson, IEEE Intelligent Systems, 28.6 (2013)

7. M. V. Sergievskiy, S. N. Syroezhkin, Cloud of science, 3.1 (2016)

8. V.A. Rustinov, A.R. Sorokin, Radioelectronics and informatics, 4 (67) (2014)

9. N.A. Verzun, M. O. Kolbanev, Omsk scientific news, 4 (148) (2016)

10. K. Won-Ho, H. Lee, N. Sanjeewa, Life Science Journal, 11.7 (2014)

11. F. D. Yu, et al., Applied Mechanics and Materials. Vol. 543 (2014)

12. U. Miroslav, J. Bienik, M. Vaculik, ELEKTRO IEEE, (2016)

13. V. Jean-Marc, et al., arXiv preprint arXiv:1602.04845 (2016)

14. J. Oliver, et al., Elektronische Sprachsignalverarbeitung. Tagungsband der 27. Konferenz, 81 (2015)

15. J. Paul, et al. "RTP Payload Format for the iSAC Codec" (2013)

16. L. Domingo, A. M. Gomez, J. L. Pérez-Córdoba, 22nd European Signal Processing Conference (EUSIPCO) (2014)

17. P. James, T. Yang, C. Lee, eds., Future Information Technology: 6th International Conference on Future Information Technology, 184 (2011)

18. D. Rutledge, "Encyclopedia of practical electronic" (2002)

19. A. Ronzhin, I. Vatamaniuk, N. Pavluk, EPE (2016)

20. I. Vatamaniuk, G. Panina, A. Saveliev, A. Ronzhin, ICARSC (2016)
21. A. Ronzhin, A. Saveliev, V. Budkov, Internet of Things, Smart Spaces, and Next Generation Networking, 307-315 (2012)

22. A. Ronzhin, A. Saveliev, O. Basov, S. Solyonyj, ICR (2016)

23. I. Vatamaniuk, D. Levonevskiy, A. Saveliev, A. Denisov, ICR (2016)

24. A. Saveliev, SPIIRAS Proc., 31, 61-79 (2013)

25. A. Saveliev, A. Ronzhin, Pattern Recognition and Image Analysis, 25.3, 517-525 (2015) 